\title{
Neu bei den Zusatzangeboten: Nachtrag zu René Schlotts Dokumentation im Januar-Heft
}

René Schlott ist im Januar-Heft der VfZ der Frage nachgegangen, warum sich wissenschaftliche Mitarbeiterinnen beziehungsweise Mitarbeiter des Instituts für Zeitgeschichte wiederholt gegen eine Übersetzung von Raul Hilbergs Pionierstudie „The Destruction of the European Jews“ ins Deutsche aussprachen. Er hat seine Dokumentation „Ablehnung und Anerkennung. Raul Hilberg und das Institut für Zeitgeschichte“ nun um zwei Dokumente ergänzt, die sich in der Rubrik „Beilagen" unserer Zusatzangebote finden. Es handelt sich um Briefe aus dem Privatarchiv des Übersetzers und Lektors Christian Seeger über die Zusammenarbeit zwischen dem Institut für Zeitgeschichte und dem Berliner Verlag Olle \& Wolter, der 1982 die erste deutsche Ausgabe von Hilbergs Pionierstudie herausgebracht hat.

www.ifz-muenchen.de/vierteljahrshefte/zusatzangebote/beilagen/ 